\title{
CHIRAL BANDS IN ${ }^{193} \mathrm{Tl}^{*}$
}

\author{
J. NDAYishimye ${ }^{a, b}$, E.A. LAWRIE ${ }^{a}$, O. Shirinda ${ }^{a, b}$, J.L. EAStON ${ }^{a, c}$ \\ S.M. WYNGAARDt ${ }^{b}$, R.A. BARK ${ }^{a}$, S.P. BVumbi ${ }^{\text {, }}$, T.R.S. DinOKO \\ P. Jones ${ }^{a}$, N.Y. Kheswa ${ }^{\text {a }}$, J.J. LAWRIE ${ }^{\mathrm{a}}$, S.N.T. MAJOlA ${ }^{\mathrm{a}, \mathrm{e}}$ \\ P.L. MASiteng ${ }^{\mathrm{d}}$, D. NeGi ${ }^{\mathrm{a}}$, J.N. ORCE ${ }^{\mathrm{c}}$, P. PAPKA ${ }^{\mathrm{b}, \mathrm{a}}$

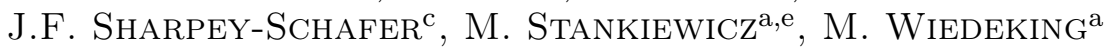

aiThemba LABS, National Research Foundation P.O. Box 722, 7129 Somerset West, South Africa

${ }^{b}$ Department of Physics, University of Stellenbosch Private Bag X1, Matieland 7602, South Africa ${ }^{\mathrm{c}}$ Department of Physics, University of the Western Cape Private Bag X17, 7535 Bellville, South Africa ${ }^{\mathrm{d}}$ Department of Physics, University of Johannesburg P.O. Box 524, 2006 Auckland Park, South Africa e Department of Physics, University of Cape Town Private Bag X3, 7701 Rondebosch, South Africa

(Received December 14, 2016)

A $\gamma$-spectroscopy study to search for chirality in ${ }^{193} \mathrm{Tl}$ was conducted. Three negative-parity bands with close excitation energies and almost equal $B(\mathrm{M} 1) / B(\mathrm{E} 2)$ values were identified. These bands were associated with the same $\pi h_{9 / 2} \otimes \nu i_{13 / 2}^{2}$ configuration which is suitable for chiral symmetry. Analysis of the properties of these bands suggested that one or two chiral systems are formed in ${ }^{193} \mathrm{Tl}$.

DOI:10.5506/APhysPolB.48.343

\section{Introduction}

Since the introduction of chirality in nuclei [1], the search for chiral candidates in different mass regions has been a hot topic for about two decades. At iThemba LABS, a new chiral symmetry region, the thallium isotopes with mass $A \geq 190$ was found. Candidate chiral bands were suggested in ${ }^{198} \mathrm{Tl}[2,3]$ and then in ${ }^{194} \mathrm{Tl}[4-6]$. Most importantly, a comparison of the properties in the chiral partner bands in ${ }^{194} \mathrm{Tl}$ showed that this nucleus is

\footnotetext{
* Presented at the Zakopane Conference on Nuclear Physics "Extremes of the Nuclear
} Landscape", Zakopane, Poland, August 28-September 4, 2016. 
one of the best chiral candidates to date [4]. A search for chiral symmetry in the neighboring thallium isotopes was undertaken. The results from a $\gamma$-ray spectroscopy study in ${ }^{193} \mathrm{Tl}$ performed at iThemba LABS, South Africa, are discussed.

\section{Experiments and data analysis}

The AFRODITE $\gamma$-ray array $[7,8]$ was used to investigate the highspin states in ${ }^{193} \mathrm{Tl}$. It comprised of 9 clovers that were mounted in two rings; 5 clovers at $90^{\circ}$ and 4 clovers at $135^{\circ}$ with respect to the beam direction. A self-supported thin target of $1.0 \mathrm{mg} / \mathrm{cm}^{2}$ was used in two reactions; ${ }^{160} \mathrm{Gd}\left({ }^{37} \mathrm{Cl}, 4 n\right)$ at $E_{\text {lab }}=167 \mathrm{MeV}$ and ${ }^{181} \mathrm{Ta}\left({ }^{18} \mathrm{O}, 6 n\right)$ at $E_{\text {lab }}=105 \mathrm{MeV}$. XIA digital electronics [9] was employed in the second experiment.

Various matrices were constructed for the data analysis. Coincidence relationships were investigated using $\gamma-\gamma$ and, where statistics allowed it, $\gamma-\gamma-\gamma$ coincidence data. Spins and parities of the states were determined in the angular distribution and linear polarization anisotropy measurements. Assuming small mixing ratios for the dipole transitions, reduced transition probability ratios $B(\mathrm{M} 1) / B(\mathrm{E} 2)$ were deduced based on the measured energies and intensities of the transitions.

\section{Results and discussion}

The analysis of the data resulted in a revision and extension of the previous level scheme [10] of ${ }^{193} \mathrm{Tl}$. A partial level scheme of ${ }^{193} \mathrm{Tl}$, as obtained in this work, is shown in Fig. 1. One band labeled Band 3 in Fig. 1, was previously placed at the positive-parity side of the level scheme, but our data showed that it is feeding the negative-parity structures. Three new linking transitions for this band were established. Another band, labeled Band 2 in Fig. 1, was previously observed. However, none of the previously suggested linking transitions of this band could be confirmed. Instead, it was connected in a different way to the negative-parity Band 1. In addition, some of the transitions in this band were placed differently. New spins and parities for the levels in Bands 2 and 3 were determined.

The three negative parity bands shown in Fig. 1 are relevant to chiral symmetry. These bands result from the coupling between an $h_{9 / 2}$ proton and two $\nu i_{13 / 2}$ neutrons. The proton has particle nature as it is located at the bottom of the $h_{9 / 2}$ shell, while the two neutrons are located at the upper part of the $i_{13 / 2}$ shell and have hole nature. Therefore, this configuration is suitable for the formation of chiral symmetry in this nucleus [1]. The three negative-parity bands in ${ }^{193} \mathrm{Tl}$ are very similar to the three chiral candidate bands in ${ }^{194} \mathrm{Tl}$ [4] that were associated with the $\pi h_{9 / 2} \otimes \nu i_{13 / 2}{ }^{3}$ configuration. 


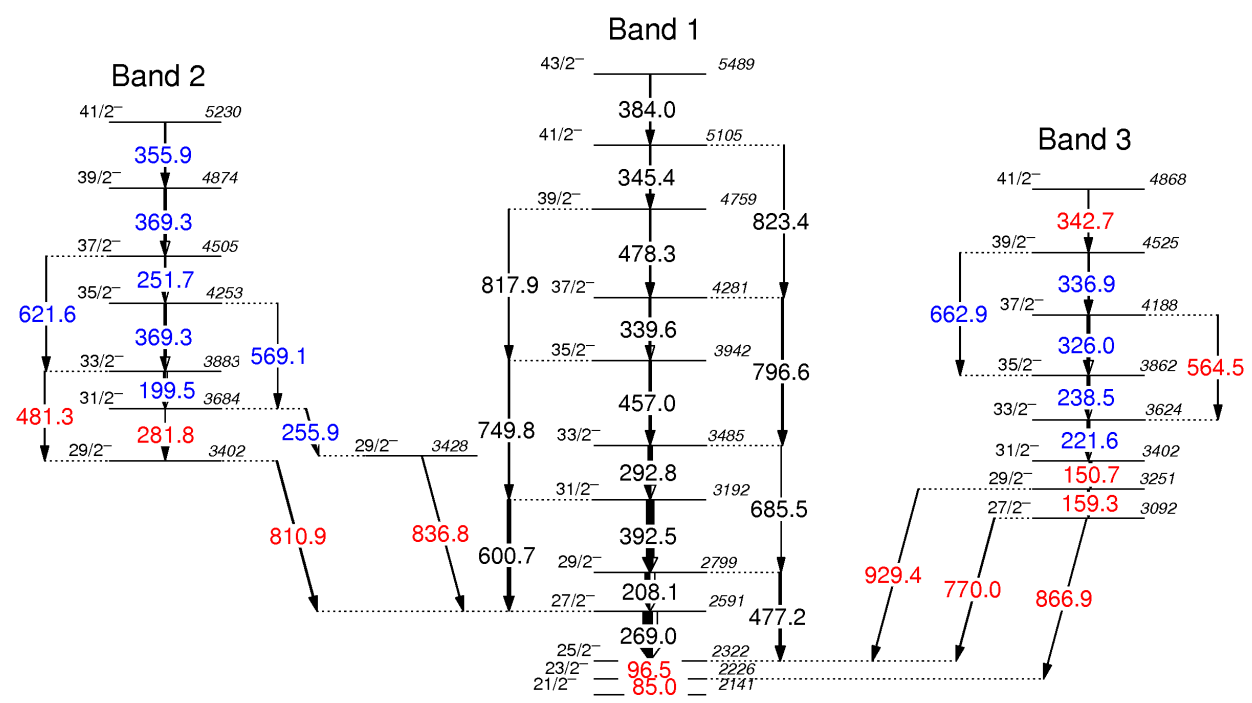

Fig. 1. (Color online) Partial level scheme showing three negative parity bands in ${ }^{193} \mathrm{Tl}$. New transitions are shown in gray/red, while the light gray/blue color indicates transitions with revised placement.

As in ${ }^{194} \mathrm{Tl}$, two of these bands have similar alignments, while the third band has an alignment of $\sim 2 \hbar$ lower. The two bands with higher alignments form the chiral pair in ${ }^{194} \mathrm{Tl}$. This pair exhibits very similar properties; the difference in the excitation energies is $\Delta E \leq 110 \mathrm{keV}$, with $\Delta E_{\min }=37 \mathrm{keV}$. The two partners also have the same alignments and $B(\mathrm{M} 1) / B(\mathrm{E} 2)$ ratios. The partner bands in ${ }^{193} \mathrm{Tl}$ with similar alignments are Bands 2 and 3 . They show similarity in the excitation energies with $\Delta E$ in the range of 151 to $362 \mathrm{keV}$. The two bands show very similar alignments and $B(\mathrm{M} 1) / B(\mathrm{E} 2)$ transition probability ratios. Thus, they are proposed as a candidate chiral pair. Band 1, associated with the same $\pi h_{9 / 2} \otimes \nu i_{13 / 2}{ }^{2}$ configuration could be a part of a second chiral symmetry structure.

Theoretical calculations were performed in order to test the suggested presence of chiral symmetry structures in ${ }^{193} \mathrm{Tl}$. Potential energy surfaces as a function of the spin were calculated using the Cranked Nilsson Strutinsky (CNS) codes [11-13]. The obtained minima indicate that the nucleus has a triaxial shape and rotates around the intermediate axis. This is in good agreement with the requirements [1] for the nuclear chiral symmetry formation. 


\section{Summary}

In summary, the performed study provides a new insight in the highspin structures of ${ }^{193} \mathrm{Tl}$. In particular, three negative-parity bands were established. They indicate that one or two chiral systems are formed in ${ }^{193} \mathrm{Tl}$. The bands exhibit similar properties as those observed in ${ }^{194} \mathrm{Tl}$. Theoretical calculations suggest that the nuclear shape of ${ }^{193} \mathrm{Tl}$ is triaxial, and thus support the presence of chiral symmetry.

We would like to thank the crew of the iThemba LABS separated sector cyclotron for the excellent beam delivery. This work is based upon research supported by the National Research Foundation, South Africa.

\section{REFERENCES}

[1] S. Frauendorf, J. Meng, Nucl. Phys. A 617, 131 (1997).

[2] E.A. Lawrie et al., Phys. Rev. C 78, 021305(R) (2008).

[3] E.A. Lawrie et al., Eur. Phys. J. A 45, 39 (2010).

[4] P.L. Masiteng et al., Phys. Lett. B 719, 83 (2013).

[5] P.L. Masiteng et al., Eur. Phys. J. A 50, 119 (2014).

[6] P.L. Masiteng et al., Eur. Phys. J. A 52, 28 (2016).

[7] R.T.N. Newman et al., Balkan Phys. Lett., Special Issue 1998, p. 182.

[8] J.F. Sharpey-Schafer, Nucl. Phys. News 14, 5 (2004).

[9] www.xia.com

[10] W. Reviol et al., Nucl. Phys. A 548, 331 (1992).

[11] T. Bengtsson, I. Ragnarsson, Nucl. Phys. A 436, 14 (1985).

[12] B.G. Carlsson, I. Ragnarsson, Phys. Rev. C 74, 011302(R) (2006).

[13] I. Ragnarsson, B.G. Carlsson, CNS Manual, 2010. 